\title{
RELATIONSHIP BETWEEN POLYNOMIALS WITH MULTIPLE ROOTS AND RATIONAL FUNCTIONS WITH COMMON ROOTS
}

\author{
YASUHIKO KAMIYAMA
}

\begin{abstract}
For $F=\mathrm{R}$ or $\mathrm{C}$, let $P_{k, n}^{l}(F)$ denote the space of monic polynomials $f(z)$ over $F$ of degree $k$ and such that the number of $n$-fold roots of $f(z)$ is at most $l$. Let $X_{k, n}^{l}(F)$ denote the space consisting of all $n$-tuples $\left(p_{1}(z), \ldots, p_{n}(z)\right)$ of monic polynomials over $F$ of degree $k$ and such that there are at most $l$ roots common to all $p_{i}(z)$. In this paper, we prove that $P_{k, n}^{l}(F)$ and $X_{\left[\frac{k}{n}\right], n}^{l}(F)$ are stably homotopy equivalent. In fact, they are homotopy equivalent when $F=\mathrm{C}$ and $(n, l) \neq(2,0)$. We also consider the case that $n$-fold roots and common roots are not real. These results generalize previous results concerning these spaces.
\end{abstract}

\section{Introduction}

The purpose of this paper is to study the relationship between the space of polynomials with multiple roots and the space of rational functions with common roots. We prove a theorem which generalizes previous results concerning these spaces.

Let $C_{k}(\mathrm{C})$ denote the configuration space of unordered $k$-tuples of distinct points in $\mathrm{C}$. The space $C_{k}(\mathrm{C})$ has some interesting connections with the theory of algebraic functions and Artin's theory of braids. For instance, $C_{k}(\mathrm{C})$ is the space of monic polynomials over $C$ of degree $k$ without multiple roots. On the other hand, we have $C_{k}(\mathrm{C})=K\left(\beta_{k}, 1\right)$, where $\beta_{k}$ is Artin's braid group on $k$-strings and $K\left(\beta_{k}, 1\right)$ is the corresponding Eilenberg-MacLane space. Thus the homology group of $C_{k}(\mathrm{C})$ has a certain significance.

In [1], Arnold proved important theorems on the integral homology of $C_{k}(\mathrm{C})$, e.g. the stability of the homology. The proofs proceed as follows. Let $P_{k, n}^{l}(\mathrm{C})$ denote the space of monic polynomials over C of degree $k$ and such that the number of $n$-fold roots is at most $l$. (Compare Definition 2.1.) (We allow $n$-fold roots to coincide. Hence, an element of the complement of $P_{k, n}^{l}(\mathrm{C})$ in $C^{k}$ is of the form $\left(g_{l+1}(z)\right)^{n} h_{k-(l+1) n}(z)$, where $g_{l+1}(z)$ and $h_{k-(l+1) n}(z)$ are arbitrary monic polynomials of degrees $l+1$ and $k-(l+1) n$, respectively.)

Received January 9, 2004. 
Let $\bar{\Sigma}_{k, n}^{l}$ denote the complement of $P_{k, n}^{l}(\mathrm{C})$ in $S^{2 k}=\mathrm{C}^{k} \cup\{\infty\}$. By induction with making $k$ larger and $l$ smaller while $n$ being fixed, one obtains information on $\bar{\Sigma}_{k, n}^{l}$ for all $k, n$ and $l$. By the Alexander duality, the information is equivalent to that on $P_{k, n}^{l}(\mathrm{C})$. In particular, setting $n=2$ and $l=0$, we obtain information on $C_{k}(\mathrm{C})$.

Later, the homology group $H_{*}\left(C_{k}(\mathrm{C}) ; \mathrm{Z} / p\right)$ was determined (using different methods) in [8] for $p=2$ and in [6] for an odd prime $p$. Using this, the stable homotopy type of $C_{k}(\mathrm{C})$ was described in [3] in terms of Snaith's stable summands of $\Omega^{2} S^{3}$.

On the other hand, Segal studied the topology of spaces of rational functions ([13]). Let Rat ${ }_{k}(n)$ denote the space consisting of all $n$-tuples $\left(p_{1}(z), \ldots\right.$, $\left.p_{n}(z)\right)$ of monic polynomials over $\mathrm{C}$ of degree $k$ and such that there are no roots common to all $p_{i}(z)$. $\operatorname{Rat}_{k}(n)$ is considered to be the space of holomorphic maps of degree $k$ from $S^{2}$ to $C P^{n-1}$ with the basepoint condition $\infty \mapsto[1, \ldots, 1]$. There is an inclusion

$$
i_{k}: \operatorname{Rat}_{k}(n) \hookrightarrow \Omega_{k}^{2} C P^{n-1} \simeq \Omega^{2} S^{2 n-1} .
$$

It is proved in [13] that $i_{k}$ is a homotopy equivalence up to dimension $k(2 n-3)$. Later, the stable homotopy type of $\operatorname{Rat}_{k}(n)$ was described (using different methods) in [4] and [5] in terms of Snaith's stable summands of $\Omega^{2} S^{2 n-1}$. In particular, we consider the case $n=2$. Combining the results of [3], [4] and [5], we obtain a stable homotopy equivalence $C_{k}(\mathrm{C}) \underset{s}{\simeq} \operatorname{Rat}_{\left[\frac{k}{2}\right]}(2)$, where $\left[\frac{k}{2}\right]$ is as usual the largest integer $\leq \frac{k}{2}$. This result was generalized in [14] as follows:

$$
P_{k, n}^{0}(\mathrm{C}) \underset{s}{\simeq} \operatorname{Rat}_{\left[\frac{k}{n}\right]}(n),
$$

where $P_{k, n}^{0}(\mathrm{C})$ is defined above. Later, (1.1) was improved to a homotopy equivalence for $n \geq 3$. (Compare (2.3).)

If we take Arnold's proof and (1.1) into account, we naturally encounter the following problem: what is the (stable) homotopy type of $P_{k, n}^{l}(\mathrm{C})$ for $k \geq 1$, $n \geq 2$ and $l \geq 0$. The purpose of this paper is to study this. In fact, we consider four cases according as whether polynomials are defined over R or C, and whether we allow $n$-fold roots or common roots to be real or not. Our main results will be stated in Section 2. (Compare Theorems A, B and C.) Here we summarize the result for the most interesting case.

TheоReм 1.2. Let $X_{k, n}^{l}(\mathrm{C})$ be the space consisting of all $n$-tuples $\left(p_{1}(z)\right.$, $\left.\ldots, p_{n}(z)\right)$ of monic polynomials over $\mathrm{C}$ of degree $k$ and such that there are at most $l$ roots common to all $p_{i}(z)$. Then 
(i) For all $k, n$ and $l, X_{k, n}^{l}(\mathrm{C})$ is stably homotopy equivalent to the $k$-th filtration of the homotopy theoretic fiber of the inclusion $J^{l}(2 n-2) \hookrightarrow$ $\Omega S^{2 n-1}$, where $J^{l}(2 n-2)$ denotes the l-th stage of the James construction which builds $\Omega S^{2 n-1}$.

(ii) Except when $(n, l)=(2,0)$, there is a homotopy equivalence

$$
P_{k, n}^{l}(\mathrm{C}) \simeq X_{\left[\frac{k}{n}\right], n}^{l}(\mathrm{C}) .
$$

Note that our information on $P_{k, n}^{l}(\mathrm{C})$ gives that on $\bar{\Sigma}_{k, n}^{l}$, since these spaces are Spanier-Whitehead dual to one another.

This paper is organized as follows. In Section 2, we state the main results. We define four kinds of spaces consisting of polynomials and four kinds of spaces consisting of $n$-tuples of polynomials. Theorem A asserts that the corresponding spaces are stably homotopy equivalent. Theorem B gives stable splittings of these spaces. Theorem $\mathrm{C}$ asserts that in the most interesting case, the stable homotopy equivalence in Theorem $\mathrm{A}$ is in fact a homotopy equivalence. In Section 3, we recall previous results about the spaces in Section 2 and see how they are contained in Theorems A, B and C. In Section 4, we prove Theorem B. In Section 5, we prove Theorem A (i) for $F=C$ and Theorem C. In Section 6, we prove Theorem A (i) for $F=\mathrm{R}$ and (ii).

\section{Main results}

Definition 2.1. For $F=\mathrm{R}$ or $\mathrm{C}$, we set

(i) $P_{k, n}^{l}(F)=\{f(z): f(z)$ is a monic polynomial over $F$ of degree $k$ and such that the number of $n$-fold roots is at most $l$.

(ii) $Q_{k, n}^{l}(F)=\left\{f(z) \in P_{k, n}^{l}(F): f(z)\right.$ has no $n$-fold roots in $\left.\mathrm{R}\right\}$.

(iii) $X_{k, n}^{l}(F)=\left\{\left(p_{1}(z), \ldots, p_{n}(z)\right)\right.$ : each $p_{i}(z)$ is a monic polynomial over $F$ of degree $k$ and such that there are at most $l$ roots common to all $\left.p_{i}(z)\right\}$.

(iv) $Y_{k, n}^{l}(F)=\left\{\left(p_{1}(z), \ldots, p_{n}(z)\right) \in X_{k, n}^{l}(F):\right.$ there are no real roots common to all $\left.p_{i}(z)\right\}$.

Remarks. 1. By definition, we have $P_{k, n}^{0}(F)=Q_{k, n}^{0}(F)$ and $X_{k, n}^{0}(F)=$ $Y_{k, n}^{0}(F)$. In particular, $X_{k, n}^{0}(\mathrm{C})=\operatorname{Rat}_{k}(n)$.

2. Note that

$$
Q_{k, n}^{2 i+1}(\mathrm{R})=Q_{k, n}^{2 i}(\mathrm{R}) \quad \text { and } \quad Y_{k, n}^{2 i+1}(\mathrm{R})=Y_{k, n}^{2 i}(\mathrm{R}),
$$


since if $\alpha \in H_{+}$(where $H_{+}$is the open upper half-plane) is a root of a polynomial over $\mathrm{R}$, then so is $\bar{\alpha} \in H_{-}$(where $\bar{\alpha}$ is the complex conjugate of $\alpha$ ).

We have the following diagrams:

$$
\begin{gathered}
Q_{k, n}^{\left[\frac{k}{n}\right]}(F) \supset Q_{k, n}^{\left[\frac{k}{n}\right]-1}(F) \supset \cdots \supset Q_{k, n}^{l}(F) \supset \cdots \supset Q_{k, n}^{0}(F) \\
\cap \quad \cap \\
P_{k, n}^{\left[\frac{k}{n}\right]}(F) \supset P_{k, n}^{\left[\frac{k}{n}\right]-1}(F) \supset \cdots \supset P_{k, n}^{l}(F) \supset \cdots \supset P_{k, n}^{0}(F)
\end{gathered}
$$

and

$$
\begin{gathered}
Y_{k, n}^{k}(F) \supset Y_{k, n}^{k-1}(F) \supset \cdots \supset Y_{k, n}^{l}(F) \supset \cdots \supset Y_{k, n}^{0}(F) \\
\cap \quad \begin{array}{c}
\cap \\
X_{k, n}^{k}(F) \supset X_{k, n}^{k-1}(F) \supset \cdots \supset X_{k, n}^{l}(F) \supset \cdots \supset X_{k, n}^{0}(F)
\end{array}
\end{gathered}
$$

where each subset is an open set.

Example 2.2. We set $d=1$ for $F=\mathrm{R}$ and $d=2$ for $F=\mathrm{C}$. Then there are homotopy equivalences

(i) $P_{k, n}^{\left[\frac{k}{n}\right]}(F) \simeq\{$ a point $\}$ and $P_{k, n}^{\left[\frac{k}{n}\right]-1}(F) \simeq S^{d\left[\frac{k}{n}\right](n-1)-1}$, where the case for $F=C$ and $(k, n)=(3,2)$ is excluded from the second homotopy equivalence.

(ii) $Q_{k, n}^{1}(F) \simeq S^{d n-2}(n \leq k<2 n)$.

(iii) $X_{k, n}^{l}(F) \simeq\{$ a point $\}(k \leq l)$ and $X_{k, n}^{k-1}(F) \simeq S^{d k(n-1)-1}$.

(iv) $Y_{1, n}^{1}(F) \simeq S^{d n-2}$.

Proof. Since the proofs are similar, we prove only the second homotopy equivalences of (i) and (iii). Let $\Sigma$ be the complement of $P_{k, n}^{\left[\frac{k}{n}\right]-1}(F)$ in $F^{k}$. A polynomial $f \in F^{k}$ (hereafter we omit the variable $z$ from each polynomial) belongs to $\Sigma$ if and only if the number of $n$-fold roots of $f$ is exactly $\left[\frac{k}{n}\right]$, hence $f$ is of the form $g_{\left[\frac{k}{n}\right]}^{n} h_{k-\left[\frac{k}{n}\right] n}$, where $g_{\left[\frac{k}{n}\right]}$ and $h_{k-\left[\frac{k}{n}\right] n}$ are arbitrary monic polynomials of degrees $\left[\frac{k}{n}\right]$ and $k-\left[\frac{k}{n}\right] n$, respectively. Thus $\Sigma \cong F^{k-\left[\frac{k}{n}\right](n-1)}$. By the Alexander duality, we obtain (i).

An element $\left(p_{1}, \ldots, p_{n}\right) \in\left(F^{k}\right)^{n}$ belongs to $X_{k, n}^{k-1}(F)$ if and only if $p_{i} \neq p_{j}$ for some $i, j$. Hence (iii) holds. This completes the proof of Example 2.2.

The homotopy types of $Q_{k, n}^{\left[\frac{k}{n}\right]}(F)$ and $Y_{k, n}^{k}(F)$ are known in [11]. (Compare (3.1) and (3.2).) Note that Example 2.2 (ii) and (iv) are special cases of them.

Our first result is the following: 
THEOREM A. For all $k, n$ and $l$, there are stable homotopy equivalences

(i) $P_{k, n}^{l}(F) \underset{s}{\simeq} X_{\left[\frac{k}{n}\right], n}^{l}(F)$.

(ii) $Q_{k, n}^{l}(F) \underset{s}{\simeq} Y_{\left[\frac{k}{n}\right], n}^{l}(F)$.

Remark. There are unstable maps $P_{k, n}^{l}(F) \rightarrow X_{k, n}^{l}(F)$ and $Q_{k, n}^{l}(F) \rightarrow$ $Y_{k, n}^{l}(F)$ defined as follows:

$$
f(z) \mapsto\left(f(z), f^{\prime}(z)+f(z), \ldots, f^{(n-1)}(z)+f(z)\right) .
$$

We can prove that these maps are homotopy equivalences as $k \rightarrow \infty$.

In [14], the result $P_{k n, n}^{0}(\mathrm{C}) \underset{s}{\simeq} X_{k, n}^{0}(\mathrm{C})$ was proved as follows. First, we prove that the complements of these spaces in $S^{2 k n}$ are stably homotopy equivalent. Next, we use the fact that the Spanier-Whitehead duals of stably homotopy equivalent spaces are stably homotopy equivalent. In contrast to this, we prove Theorem A by constructing stable maps from the right-hand sides to the lefthand sides along the lines of [5] and [10]. (Compare (B) of Sections 5 and 6.)

Next, we give stable splittings of $X_{k, n}^{l}(F)$ and $Y_{k, n}^{l}(F)$. For that purpose, we prepare some notations. (Compare [10] or Section 5.) Let $J^{l}(2 n-2)$ denote the $l$-th stage of the James construction which builds $\Omega S^{2 n-1}$, and let $W^{l}(n)$ be the homotopy theoretic fiber of the inclusion $J^{l}(2 n-2) \hookrightarrow \Omega S^{2 n-1}$. The May-Milgram model for $\Omega^{2} S^{2 n-1}$ is generalized to construct a space $\xi^{l}(n)$ combinatorially so that $\xi^{l}(n) \simeq W^{l}(n) . \xi^{l}(n)$ has a filtration $\left\{F_{q} \xi^{l}(n)\right\}$ and we set $D_{q} \xi^{l}(n)=F_{q} \xi^{l}(n) / F_{q-1} \xi^{l}(n)$. Then we have a stable splitting

$$
W^{l}(n) \underset{s}{\simeq} \bigvee_{1 \leq q} D_{q} \xi^{l}(n),
$$

which is a generalization of Snaith's stable splitting of $\Omega^{2} S^{2 n-1}$ for $l=0$.

THEOREM B. There are stable homotopy equivalences

(i) $X_{k, n}^{l}(\mathrm{C}) \simeq \bigvee_{q=1}^{k} D_{q} \xi^{l}(n)$.

(ii) $X_{k, n}^{2 i+1}(\mathrm{R}) \simeq X_{\left[\frac{k}{2}\right], n}^{i}(\mathrm{C})$, and

$X_{k, n}^{2 i}(\mathrm{R}) \underset{s}{\simeq} X_{\left[\frac{k}{2}\right], n}^{i}(\mathrm{C}) \vee \Sigma^{2 i(n-1)}\left(\bigvee_{\substack{p+2 q \leq k-2 i \\ 1 \leq p}} \Sigma^{p(n-2)} D_{q} \xi^{0}(n) \vee \bigvee_{p=1}^{k-2 i} S^{p(n-2)}\right)$

(iii) $Y_{k, n}^{l}(\mathrm{C}) \underset{s}{\simeq} X_{k, n}^{l}(\mathrm{C}) \vee \bigvee_{q=1}^{l} \Sigma^{2 q(n-1)}\left(X_{k-q, n}^{l-q}(\mathrm{C}) \vee S^{0}\right), 0 \leq l \leq k$. 
(iv) $Y_{k, n}^{2 i}(\mathrm{R}) \underset{s}{\simeq} \bigvee_{p+2 q \leq k} \Sigma^{p(n-2)} D_{q} \xi^{i}(n) \vee \bigvee_{p=1}^{k} S^{p(n-2)}$.

Remark. The first stable homotopy equivalence of Theorem B (ii) is in fact a homotopy equivalence: $X_{k, n}^{2 i+1}(\mathrm{R}) \simeq X_{\left[\frac{k}{2}\right], n}^{i}(\mathrm{C})$.

The following theorem is a stronger version of Theorem A (i) for $F=\mathrm{C}$.

Theorem C. Except when $(n, l)=(2,0)$, there are homotopy equivalences

$$
P_{k, n}^{l}(\mathrm{C}) \simeq F_{\left[\frac{k}{n}\right]} \xi^{l}(n) \simeq X_{\left[\frac{k}{n}\right], n}^{l}(\mathrm{C}),
$$

where $F_{\left[\frac{k}{n}\right]} \xi^{l}(n)$ is a filtration of $\xi^{l}(n)$.

Remark. When $l=0$, Theorem $\mathrm{C}$ is known in [7] and [9]:

$$
P_{k, n}^{0}(\mathrm{C}) \simeq F_{\left[\frac{k}{n}\right]} \xi^{0}(n) \simeq X_{\left[\frac{k}{n}\right], n}^{0}(\mathrm{C}) \quad(n \geq 3) .
$$

\section{Previous results}

Theorem A has been studied for special cases and for these cases there are natural maps from the $n$-tuples of polynomials to certain loop spaces. We recall the known results below.

(i) As in Section 1, there is an inclusion

$$
i_{k}: X_{k, n}^{0}(\mathrm{C}) \hookrightarrow \Omega_{k}^{2} \mathrm{C} P^{n-1} \simeq \Omega^{2} S^{2 n-1} .
$$

(1.1) is same as $P_{k, n}^{0}(\mathrm{C}) \underset{s}{\simeq} X_{\left[\frac{k}{n}\right], n}^{0}(\mathrm{C})$. The first homotopy equivalence of (2.3) is proved in [9] and the second is proved in [7]. The condition $n \geq 3$ in (2.3) implies that each space is simply connected. But (2.3) does not hold for $n=2$. In fact, the facts that $\pi_{1}\left(P_{k, 2}^{0}(\mathrm{C})\right) \cong \beta_{k}$ and $\pi_{1}\left(X_{\left[\frac{k}{2}\right], 2}^{0}(\mathrm{C})\right) \cong \mathrm{Z}$ (compare [13]) imply that $P_{k, 2}^{0}(\mathrm{C}) \not X_{\left[\frac{k}{2}\right], 2}^{0}(\mathrm{C})$ for $k \geq 3$. It is also shown in [7] that $F_{2} \xi^{0}(2) \not X_{2,2}^{0}(\mathrm{C})$.

Theorem B (i) is proved in [4] and [5] for $l=0$, and in [10] for general $l$.

(ii) Let $\operatorname{Map}_{k}^{T}\left(\mathrm{C} P^{1}, C P^{n-1}\right)$ be the space of continuous basepoint-preserving conjugation-equivariant maps of degree $k$ from $C P^{1}$ to $C P^{n-1}$. There is an inclusion

$$
i_{k}: X_{k, n}^{0}(\mathrm{R}) \hookrightarrow \operatorname{Map}_{k}^{T}\left(\mathrm{C} P^{1}, \mathrm{C} P^{n-1}\right) \simeq \Omega S^{n-1} \times \Omega^{2} S^{2 n-1} .
$$

As far as the author knows, there is no published matter which proves the fact that $P_{k, n}^{0}(\mathrm{R}) \underset{s}{\simeq} X_{\left[\frac{k}{n}\right], n}^{0}(\mathrm{R})$. By the same reason as in (i), it seems likely that this 
is in fact a homotopy equivalence for $n \geq 4$. In connection with this, we have

$$
P_{k, 2}^{0}(\mathrm{R}) \simeq \coprod_{q=0}^{\left[\frac{k}{2}\right]} C_{q}(\mathrm{C}) \quad \text { and } \quad X_{\left[\frac{k}{2}\right], 2}^{0}(\mathrm{R}) \simeq \coprod_{q=0}^{\left[\frac{k}{2}\right]} X_{\min \left(q,\left[\frac{k}{2}\right]-q\right), 2}^{0}(\mathrm{C}) .
$$

The first homotopy equivalence is clear and the second is proved in [13]. Hence, $P_{k, 2}^{0}(\mathrm{R})$ and $X_{\left[\frac{k}{2}\right], 2}^{0}(\mathrm{R})$ are not unstably homotopy equivalent.

(iii) Note that $Q_{k, n}^{\left[\frac{k}{n}\right]}(F)$ is the space of polynomials without $n$-fold real roots, and $Y_{k, n}^{k}(F)$ is the space of $n$-tuples of polynomials without real common roots. Restricting to the real line, there is a natural map

$$
i_{k}: Y_{k, n}^{k}(\mathrm{C}) \rightarrow \Omega S^{2 n-1} .
$$

It is proved in [11] that there are homotopy equivalences

$$
Q_{k, n}^{\left[\frac{k}{n}\right]}(\mathrm{C}) \simeq J^{\left[\frac{k}{n}\right]}(2 n-2) \simeq Y_{\left[\frac{k}{n}\right], n}^{\left[\frac{k}{n}\right]}(\mathrm{C}) \quad(n \geq 2),
$$

where as in Section $2, J^{\left[\frac{k}{n}\right]}(2 n-2)$ denotes the $\left[\frac{k}{n}\right]$-th stage of the James construction which builds $\Omega S^{2 n-1}$.

(iv) As in (iii), there is a natural map

$$
i_{k}: Y_{k, n}^{k}(\mathrm{R}) \rightarrow \Omega S^{n-1} .
$$

It is proved in [14] that there is a homotopy equivalence

$$
Q_{k, n}^{\left[\frac{k}{n}\right]}(\mathrm{R}) \simeq J^{\left[\frac{k}{n}\right]}(n-2) \simeq Y_{\left[\frac{k}{n}\right], n}^{\left[\frac{k}{n}\right]}(\mathrm{R}) \quad(n \geq 4) .
$$

By [14, p. 88], (3.2) does not hold for $n=3$ (i.e. for $k=6,7$ or $8, Q_{k, 3}^{2}(\mathrm{R}) \not$ $\left.J^{2}(1)\right)$. But we have

$$
Q_{k, 2}^{\left[\frac{k}{2}\right]}(\mathrm{R}) \simeq\left\{\left(\left[\frac{k}{2}\right]+1\right) \text { points }\right\} \simeq Y_{\left[\frac{k}{2}\right], 2}^{\left[\frac{k}{2}\right]}(\mathrm{R}) .
$$

The first homotopy equivalence is clear and the second is proved in [12].

When $n=2$, Theorem B (iv) holds unstably:

$$
Y_{k, 2}^{2 i}(\mathrm{R}) \simeq \coprod_{q=0}^{k} X_{\min (q, k-q), 2}^{i}(\mathrm{C})
$$

Setting $i=0$ or $i=\infty$ in the homotopy equivalence, we obtain the result for $X_{\left[\frac{k}{2}\right], 2}^{0}(\mathrm{R})$ in (ii) or the result for $Y_{\left[\frac{k}{2}\right], 2}^{\left[\frac{k}{2}\right]}(\mathrm{R})$ in (iv). 
In [1], Arnold performed calculations of $H_{*}\left(P_{2 k+i, 2}^{k-1}(\mathrm{C}) ; \mathrm{Z}\right)(i \geq 0)$ in low dimensions. The results are given as follows (compare [1, p. 48]).

(1) For $1 \leq q \leq 2 k-2, H_{q}\left(P_{2 k+i, 2}^{k-1}(\mathrm{C})\right.$; Z) $=0$.

(2) For $2 k-1 \leq q \leq 2 k+3, H_{q}\left(P_{2 k+i, 2}^{k-1}(\mathrm{C})\right.$; Z) are cyclic and the orders are given by the following table.

TABLE 1. The orders of the groups $H_{q}\left(P_{2 k+i, 2}^{k-1}(\mathrm{C}) ; \mathrm{Z}\right)(2 k-1 \leq q \leq 2 k+3)$

\begin{tabular}{cccccc}
\hline$i \backslash q$ & $2 k-1$ & $2 k$ & $2 k+1$ & $2 k+2$ & $2 k+3$ \\
\hline 0,1 & $\infty$ & 0 & 0 & 0 & 0 \\
2,3 & $\infty$ & $k+1$ & 0 & 0 & 0 \\
4,5 & $\infty$ & $k+1$ & $2 / k$ & $(k+2) / 2$ & 0 \\
6,7 & $\infty$ & $k+1$ & $2 / k$ & $((k+2) / 2)(2 / k)$ & $3 / k$ \\
8,9 & $\infty$ & $k+1$ & $2 / k$ & $((k+2) / 2)(2 / k)$ & $6 / k v$ \\
$\vdots$ & $\vdots$ & $\vdots$ & $\vdots$ & $\vdots$ & $\vdots$ \\
$\infty$ & $\infty$ & $k+1$ & $2 / k$ & $((k+2) / 2)(2 / k)$ & $6 / k v$ \\
\hline
\end{tabular}

Here

(1) We introduce the notation

$$
a / b=\frac{a}{\operatorname{gcd}(a, b)},
$$

where $\operatorname{gcd}(a, b)$ is the greatest common divisor of the integers $a$ and $b$.

(2) We have $v=1$ if $k \neq \equiv 1(\bmod 4)$. If $k \equiv 1(\bmod 4)$, then $v$ is either 1 or 2. But the exact value is left unknown.

The stability theorem in [1, p. 43] is stated as follows: For a fixed $q$, $H_{q}\left(P_{2 k+i, 2}^{k-1}(\mathrm{C}) ; \mathrm{Z}\right)$ is stable for $i \geq 2(q-2 k+1)$.

Using Theorems $\mathrm{B}$ and $\mathrm{C}$, we can calculate Table 1 easily as follows. First, by Theorem C, we can replace $P_{2 k+i, 2}^{k-1}(\mathrm{C})$ by $X_{k+\left[\frac{i}{2}\right], 2}^{k-1}(\mathrm{C})$. Next, using Theorem B (i), we see that as a vector space, $H_{*}\left(X_{k+\left[\frac{i}{2}\right], 2}^{k-1}(\mathrm{C}) ; \mathrm{Z} / p\right.$ ) (where $p$ is a prime) is isomorphic to the subspace of $H_{*}\left(W^{k-1}(2) ; Z / p\right)$ spanned by monomials of weight $\leq k+\left[\frac{i}{2}\right]$. (Hence, the above stability theorem is equivalent to the following assertion, which can be proved easily: each element of $H_{q}\left(W^{k-1}(2) ; \mathbf{Z} / p\right)$ has weight $\leq q-k+1$.) It is easy to determine $H_{*}\left(W^{k-1}(2) ; Z / p\right)$ from the mod $p$ Serre spectral sequence for the fibration

$$
\Omega^{2} S^{3} \rightarrow W^{k-1}(2) \rightarrow J^{k-1}(2) .
$$


The result is given in [10]. As an application, we can determine the value of $v$.

Lemma 3.3. In Table 1 , we have $v=1$ if $k \equiv 1(\bmod 4)$.

Proof. We set $k=4 s+1$. Since $6 / k v$ is in the stable range, it suffices to determine $H_{8 s+5}\left(W^{4 s}(2) ; Z\right)$. Since $6 / k v=\left\{\begin{array}{ll}\text { even } & v=1 \\ \text { odd } & v=2\end{array}\right.$ and $2 / k=2$, we have

$$
H_{8 s+5}\left(W^{4 s}(2) ; \mathrm{Z} / 2\right) \cong \begin{cases}\mathrm{Z} / 2 \oplus \mathrm{Z} / 2 & v=1 \\ \mathrm{Z} / 2 & v=2 .\end{cases}
$$

From the mod 2 Serre spectral sequence for the fibration $\Omega^{2} S^{3} \rightarrow W^{4 s}(2) \rightarrow$ $J^{4 s}(2)$, we have

$$
E_{p, *}^{8} \cong \begin{cases}x^{4 i} \otimes \frac{H_{*}\left(\Omega^{2} S^{3} ; \mathrm{Z} / 2\right)}{\left(\iota, Q_{1}(\iota)\right)} & p=8 i \text { with } 0 \leq i \leq s-1 \\ x^{4 s} \otimes H_{*}\left(\Omega^{2} S^{3} ; \mathrm{Z} / 2\right) & p=8 s \\ 0 & \text { otherwise }\end{cases}
$$

where $x$ and $\iota$ are (torsion free) generators of $H_{2}\left(\Omega S^{3} ; Z / 2\right)$ and $H_{1}\left(\Omega^{2} S^{3} ; Z / 2\right)$, respectively, and $\left(\iota, Q_{1}(\iota)\right)$ is the ideal generated by $\iota$ and $Q_{1}(\iota)$. It is clear that $x^{4 s} \otimes \iota^{5}$ and $x^{4 s} \otimes \iota^{2} * Q_{1}(\iota)$ are nonzero permanent cycles. Hence, $H_{8 s+5}\left(W^{4 s}(2) ; \mathrm{Z} / 2\right) \cong \mathrm{Z} / 2 \oplus \mathrm{Z} / 2$. This completes the proof of Lemma 3.3.

\section{Proof of Theorem B}

In this section, every homology is with $\mathrm{Z} / p$-coefficients, where $p$ is a prime. Theorem B (i) is proved in [10]. Since the proofs of (ii)-(iv) are similar, we prove (ii).

Proposition 4.1. The homologies of the both sides of Theorem B (ii) are isomorphic.

Proof. We prove by induction with making $l$ larger. The case for $l=0$ is proved as follows. First, by constructing homology classes explicitly, we find a lower bound for the mod $p$ homology of $X_{k, n}^{0}(\mathrm{R})$. (Compare Lemma 4.2.) Next, considering a geometrical resolution of a resultant, we construct a spectral sequence of the Vassiliev type. The spectral sequence converges to the mod $p$ homology of $X_{k, n}^{0}(\mathrm{C})$ and the $E^{1}$-term coincides with the lower bound. Hence, the spectral sequence collapses at the $E^{1}$-term and the lower bound is in fact an upper bound. (Compare Lemma 4.4.)

LEMMA 4.2. We define the weight of an element of $H_{*}\left(\Omega S^{n-1}\right)$ as usual, but that of $H_{*}\left(\Omega^{2} S^{2 n-1}\right)$ to be twice the usual one. Let $L_{k}$ be the subspace 
of $H_{*}\left(\Omega S^{n-1} \times \Omega^{2} S^{2 n-1}\right)$ spanned by monomials of weight $\leq k$. Then every element of $L_{k}$ is in the image of $i_{k *}$, where $i_{k}: X_{k, n}^{0}(\mathrm{R}) \hookrightarrow \Omega S^{n-1} \times \Omega^{2} S^{2 n-1}$ is the inclusion. Hence, these elements are a lower bound for $H_{*}\left(X_{k, n}^{0}(\mathrm{R})\right)$.

Proof. The proof of the lemma is similar to [2] and proceeds as follows. First, there is an inclusion

$$
\eta_{q, i, n}: X_{q, n}^{i}(\mathrm{C}) \hookrightarrow X_{2 q, n}^{2 i}(\mathrm{R}) .
$$

To construct this, we fix a homeomorphism $h: \mathrm{C} \cong H_{+}$. For $\left(p_{1}(z), \ldots\right.$, $\left.p_{n}(z)\right) \in X_{q, n}^{i}(\mathrm{C})$, we write $p_{j}(z)=\prod_{s=1}^{q}\left(z-\alpha_{s, j}\right)$. Then we set

$$
\begin{aligned}
\eta_{q, i, n} & \left(p_{1}(z), \ldots, p_{n}(z)\right) \\
& =\left(\prod_{s=1}^{q}\left(z-h\left(\alpha_{s, 1}\right)\right)\left(z-\overline{h\left(\alpha_{s, 1}\right)}\right), \ldots, \prod_{s=1}^{q}\left(z-h\left(\alpha_{s, n}\right)\right)\left(z-\overline{h\left(\alpha_{s, n}\right)}\right)\right) .
\end{aligned}
$$

Take an element $\alpha \otimes \beta \in H_{*}\left(\Omega S^{n-1} \times \Omega^{2} S^{2 n-1}\right)$ of weight $\leq k$. By [2], we can construct $\beta$ in $X_{q, n}^{0}(\mathrm{C})$ for some $q$, hence using $\eta_{q, 0, n}$, we can construct $\beta$ in $X_{2 q, n}^{0}(\mathrm{R})$. Then using the loop sum $\mu: X_{k_{1}, n}^{0}(\mathrm{R}) \times X_{k_{2}, n}^{0}(\mathrm{R}) \rightarrow X_{k_{1}+k_{2}, n}^{0}(\mathrm{R})$, which is defined in the same way as in the loop sum $X_{k_{1}, n}^{0}(\mathrm{C}) \times X_{k_{2}, n}^{0}(\mathrm{C}) \rightarrow$ $X_{k_{1}+k_{2}, n}^{0}(\mathrm{C})$ in [2], we can construct $\alpha \otimes \beta$ in $X_{k, n}^{0}(\mathrm{R})$. This completes the proof of Lemma 4.2.

\section{LEMMA 4.4. The lower bound of Lemma 4.2 is in fact an upper bound.}

Proof. We prove the lemma along the lines of [14, p. 151]. We indicate where to change. Let $\bar{\Sigma}$ be the complement of $X_{k, n}^{0}(\mathrm{R})$ in $S^{k}=\mathrm{R}^{k} \cup\{\infty\}$. There is a space $G(\bar{\Sigma})$, a geometrical resolution of $\bar{\Sigma}$, so that $G(\bar{\Sigma}) \simeq \bar{\Sigma}$. $G(\bar{\Sigma})$ has a filtration

$$
F_{0}(G(\bar{\Sigma}))=\{\infty\} \subset F_{1}(G(\bar{\Sigma})) \subset F_{2}(G(\bar{\Sigma})) \subset \cdots \subset F_{k}(G(\bar{\Sigma}))=G(\bar{\Sigma}) .
$$

$F_{p}(G(\bar{\Sigma}))-F_{p-1}(G(\bar{\Sigma}))$ has connected components indexed by non-negative integers $(s, t)$ with $s+2 t=p$. If we forget the "simplex part", then $F_{p}(G(\bar{\Sigma}))-$ $F_{p-1}(G(\bar{\Sigma}))$ consists of $n$-tuples of polynomials which have exactly $p$ distinct common roots. The integers $(s, t)$ with $s+2 t=p$ parametrize the connected component in which $s$ of the common roots are real and $t$ of them belong to $H_{+}$(where $H_{+}$is the open upper half-plane), hence $t$ of them belong to $H_{-}$since polynomials are real. The connected component parametrized by $(s, t)$ is a fibered product of the following two fiber bundles: They have a common base $C_{s}(\mathrm{R}) \times C_{t}\left(H_{+}\right)$, where $C_{i}(X)$ denotes the configuration space of unordered $i$-tuples of distinct points in $X$. The fibers of the two bundles 
are the $(s+t-1)$-dimensional open simplex and $\mathbf{R}^{(k-p) n}$, respectively. Let $E^{r}(G(\bar{\Sigma}))$ be the mod $p$ spectral sequence associated to the filtration (4.5). The above argument shows that $E_{p, q}^{1}$ may be nontrivial only for $1 \leq p \leq k$ so that

$$
E_{p, q}^{1}=\bigoplus_{s+2 t=p} H^{(k-p) n+s+t-q-1}\left(C_{t}(\mathrm{C}) ; \pm \mathrm{Z} / p\right),
$$

where $\pm \mathbf{Z}$ / $p$ denotes the local system locally isomorphic to $\mathbf{Z} / p$ but changes the orientation over the loops defining odd permutations. Using the fact that $D_{t} \xi^{0}(n) \simeq \Sigma^{2 t(n-2)} D_{t} \xi^{0}(2)$ (compare [3]), this is equivalent to

$$
E_{p, q}^{1}=\bigoplus_{t=1}^{\left[\frac{p}{2}\right]} \widetilde{H}^{k n+2 t(n-2)-p(n-1)-q-1}\left(D_{t} \xi^{0}(n)\right) \oplus \widetilde{H}^{k n-p(n-1)-q-1}\left(S^{0}\right) .
$$

Let $1 \leq *$. From the Alexander duality, we have $\operatorname{dim} H_{*}\left(X_{k, n}^{0}(\mathrm{R})\right)$

$$
\leq \sum_{p=2}^{k} \sum_{t=1}^{\left[\frac{p}{2}\right]} \operatorname{dim} H_{*}\left(\Sigma^{(p-2 t)(n-2)} D_{t} \xi^{0}(n)\right)+\sum_{p=1}^{k} \operatorname{dim} H_{*}\left(S^{p(n-2)}\right) .
$$

Let $u_{n-2}$ be the generator of $H_{n-2}\left(\Omega S^{n-1}\right)$. Identifying $H_{*}\left(\Sigma^{(p-2 t)(n-2)}\right.$ $\left.D_{t} \xi^{0}(n)\right)$ with $u_{n-2}^{p-2 t} \otimes \widetilde{H}_{*}\left(D_{t} \xi^{0}(n)\right)$ and $H_{*}\left(S^{p(n-2)}\right)$ with $u_{n-2}^{p}$, we see that $H_{*}\left(X_{k, n}^{0}(\mathrm{R})\right)$ is at most as big as $L_{k}$. This completes the proof of Lemma 4.4, and, consequently, Proposition 4.1 holds for $l=0$.

Next, we prove Proposition 4.1 for general $l$. By the same arguments as in [10, Propositions 4.5, 5.4], we have the following long exact sequence:

$$
\begin{aligned}
\cdots \longrightarrow H_{*}\left(X_{k, n}^{l-1}(\mathrm{R})\right) \stackrel{i_{*}}{\longrightarrow} H_{*}\left(X_{k, n}^{l}(\mathrm{R})\right) & \\
& \longrightarrow H_{*-l(n-1)}\left(X_{k-l, n}^{0}(\mathrm{R})\right) \stackrel{\Phi}{\longrightarrow} H_{*-1}\left(X_{k, n}^{l-1}(\mathrm{R})\right) \longrightarrow \cdots
\end{aligned}
$$

where $i$ is the inclusion. To construct this, note the following decomposition as sets

$$
X_{k, n}^{l}(\mathrm{R})-X_{k, n}^{l-1}(\mathrm{R})=\coprod_{s+2 t=l} \operatorname{SP}^{s}(\mathrm{R}) \times \operatorname{SP}^{t}\left(H_{+}\right) \times X_{k-l, n}^{0}(\mathrm{R})
$$

and use the fact that $H_{c}^{*}\left(\operatorname{SP}^{s}(\mathrm{R})\right)=0$ for $s \geq 2$, where $H_{c}^{*}$ is the cohomology with compact supports.

The homomorphism

$$
\Phi: H_{*-l(n-1)}\left(X_{k-l, n}^{0}(\mathrm{R})\right) \longrightarrow H_{*-1}\left(X_{k, n}^{l-1}(\mathrm{R})\right)
$$


is given as follows. We give the case for $l=2 i$. It is easy to see that the second homotopy equivalence of Theorem B (ii) is equivalent to

$$
X_{k, n}^{2 i}(\mathrm{R}) \underset{s}{\simeq} X_{\left[\frac{k}{2}\right], n}^{i}(\mathrm{C}) \vee \Sigma^{(2 i+1)(n-1)-1}\left(X_{k-2 i-1, n}^{0}(\mathrm{R}) \vee S^{0}\right) .
$$

From inductive hypothesis, we have

$$
\begin{aligned}
& H_{*-2 i(n-1)}\left(X_{k-2 i, n}^{0}(\mathrm{R})\right) \\
& \quad \cong H_{*-2 i(n-1)}\left(X_{\left[\frac{k}{2}\right]-i, n}^{0}(\mathrm{C})\right) \oplus \widetilde{H}_{*-2 i(n-1)}\left(\Sigma^{n-2} X_{k-2 i-1, n}^{0}(\mathrm{R}) \vee S^{n-2}\right)
\end{aligned}
$$

and

$$
H_{*-1}\left(X_{k, n}^{2 i-1}(\mathrm{R})\right) \cong H_{*-1}\left(X_{\left[\frac{k}{2}\right], n}^{i-1}(\mathrm{C})\right)
$$

Let

$$
\psi: H_{*-2 i(n-1)}\left(X_{\left[\frac{k}{2}\right]-i, n}^{0}(\mathrm{C})\right) \longrightarrow H_{*-1}\left(X_{\left[\frac{k}{2}\right], n}^{i-1}(\mathrm{C})\right)
$$

be the homomorphism corresponding to $\Phi$ in the long exact sequence (4.6) with $X(\mathrm{R})$ replaced by $X(\mathrm{C})$. Then $\Phi:(4.8) \rightarrow(4.9)$ is given by mapping the first summand by $\psi$ and the second summand by 0 . Hence Proposition 4.1 holds for general $l$.

Finally, we construct (stable) maps from the right-hand side of Theorem B (ii). First, the unstable map from the right-hand side of the first homotopy equivalence of Theorem B (ii) or the first stable summand in (4.7) is essentially the inclusion $\eta_{q, i, n}$ in (4.3). Next, the stable map from the second stable summand in (4.7) is constructed as follows. By Example 2.2 (iii), we have an inclusion $\iota: S^{(2 i+1)(n-1)-1} \hookrightarrow X_{2 i+1, n}^{2 i}(\mathrm{R})$. Consider the following composite of maps

$$
\begin{aligned}
S^{(2 i+1)(n-1)-1} \times\left(X_{k-2 i-1, n}^{0}(\mathrm{R})\right. & \left.\vee S^{0}\right) \\
& \stackrel{\iota \times 1}{\longrightarrow} X_{2 i+1, n}^{2 i}(\mathrm{R}) \times X_{k-2 i-1, n}^{0}(\mathrm{R}) \stackrel{\mu}{\longrightarrow} X_{k, n}^{2 i}(\mathrm{R}),
\end{aligned}
$$

where $\mu$ is the loop sum. This induces a stable map from the second stable summand in (4.7). Note that the unstable (resp. stable) map for the first (resp. second) homotopy equivalence of Theorem B (ii) are compatible with the homology splitting by weights. Using Proposition 4.1, it is easy to show that these maps induce isomorphisms in homology, hence are stable homotopy equivalences. This completes the proofs of Theorems B (ii).

\section{Proofs of Theorem $\mathrm{A}$ (i) for $\boldsymbol{F}=\mathrm{C}$ and Theorem $\mathrm{C}$}

The proof of Theorem A proceeds as follows. 
(A) For $0 \leq j \leq n-1$, the inclusions

$$
P_{k n, n}^{l}(F) \hookrightarrow P_{k n+j, n}^{l}(F) \quad \text { and } \quad Q_{k n, n}^{l}(F) \hookrightarrow Q_{k n+j, n}^{l}(F)
$$

induce isomorphisms in homology. Hence, we consider $P_{k n, n}^{l}(F)$ or $Q_{k n, n}^{l}(F)$ instead of $P_{k, n}^{l}(F)$ or $Q_{k, n}^{l}(F)$.

(B) We construct stable maps

$$
f_{k, n}^{l}(F): X_{k, n}^{l}(F) \rightarrow P_{k n, n}^{l}(F) \quad \text { and } \quad g_{k, n}^{l}(F): Y_{k, n}^{l}(F) \rightarrow Q_{k n, n}^{l}(F)
$$

so that $f_{k, n}^{0}(F)=g_{k, n}^{0}(F)$. Here we consider the domains $X_{k, n}^{l}(F)$ and $Y_{k, n}^{l}(F)$ to be the right-hand sides of the stable homotopy equivalences in Theorem B. (Compare (5.2) for the construction of $f_{k, n}^{l}(\mathrm{C})$.)

(C) We prove that $f_{k, n}^{0}(F)$ is a stable homotopy equivalence.

By (1.1), we know $P_{k n, n}^{0}(\mathrm{C}) \simeq X_{k, n}^{0}(\mathrm{C})$ without specifying a stable map. Hence, for $f_{k, n}^{0}(\mathrm{C})$, the problem is only to prove that the stable homotopy equivalence is given by $f_{k, n}^{0}(\mathrm{C})$. But this is immediate from the construction of a stable map.

On the other hand, for $f_{k, n}^{0}(\mathrm{R})$, we need to prove that $P_{k n, n}^{0}(\mathrm{R}) \underset{\bar{s}}{\simeq} X_{k, n}^{0}(\mathrm{R})$ at least without specifying a map.

(D) We prove inductively that $f_{k, n}^{l}(F)$ and $g_{k, n}^{l}(F)$ are stable homotopy equivalences.

We prove Step (D) by induction with making $l$ larger.

(i) Since $f_{k, n}^{0}(F)=g_{k, n}^{0}(F)$, the initial steps for inductions are proved in Step (C).

(ii) In order to prove the case for $f_{k, n}^{l}(F)$, we construct two long exact sequences. One is to calculate $H_{*}\left(X_{k, n}^{l}(F)\right)$ from $H_{*}\left(X_{k, n}^{l-1}(F)\right)$ and $H_{*}\left(X_{k-l, n}^{0}(F)\right)$, and the other is to calculate $H_{*}\left(P_{k n, n}^{l}(F)\right)$ from $H_{*}\left(P_{k n, n}^{l-1}(F)\right)$ and $H_{*}\left(P_{(k-l) n, n}^{0}(F)\right)$ (where homology is with $\mathrm{Z}$ coefficients). These exact sequences are connected by $f_{k, n}^{l}(F), f_{k, n}^{l-1}(F)$ and $f_{k-l, n}^{0}(F)$. Hence we can perform inductions.

The case for $g_{k, n}^{l}(F)$ is proved similarly.

Theorem $\mathrm{C}$ is proved by constructing unstable maps $F_{k} \xi^{l}(n) \rightarrow P_{k n, n}^{l}(\mathrm{C})$ and $F_{k} \xi^{l}(n) \rightarrow X_{k, n}^{l}(\mathrm{C})$ which are homotopy equivalences. The arguments are refinements of the construction of the map $f_{k, n}^{l}(\mathrm{C})$ in (5.2). (Compare Proposition 5.4.)

In this section, we prove Theorem A (i) for $F=\mathrm{C}$ and Theorem C.

Proof of Theorem A (i) For $F=$ C. We follow Steps (A)-(D) above. 
(A) By the Alexander duality, the assertion of (A) is equivalent to the repetition theorem in [1, p. 42].

(B) We construct a stable map $f_{k, n}^{l}$ (C) along the lines of [5] and [10]. We recall the stable splitting $W^{l}(n) \simeq \bigvee_{1 \leq q} D_{q} \xi^{l}(n)$ in Section 2. (Compare [10, $\S 2]$.) Let $\mathscr{C}_{2}(q)$ be the set of $q$-tuples $\left\langle c_{1}, \ldots, c_{q}\right\rangle$ of little 2-cubes which are pairwise non-overlapping. For a unit disc $D^{2 n-2}$, we take a basepoint equal to $(1,0, \ldots, 0)$. Define $\Xi_{q}^{l}(n)$ to be the subspace of $\mathscr{C}_{2}(q) \times{ }_{\Sigma_{q}}\left(D^{2 n-2}\right)^{q}$ consisting of all elements of the form $\left(\left\langle c_{1}, \ldots, c_{q}\right\rangle, y_{1}, \ldots, y_{q}\right)$ such that there are at most $l$ of $y_{1}, \ldots, y_{q}$ belong to $D^{2 n-2}-S^{2 n-3}$. We define $\xi^{l}(n)$ by

$$
\xi^{l}(n)=\coprod_{0 \leq q} \Xi_{q}^{l}(n) / \approx
$$

Here

$$
\left(\left\langle c_{1}, \ldots, c_{q}\right\rangle, y_{1}, \ldots, y_{q}\right) \approx\left(\left\langle c_{1}, \ldots, \widehat{c}_{i}, \ldots, c_{q}\right\rangle, y_{1}, \ldots, \widehat{y}_{i}, \ldots, y_{q}\right)
$$

if $y_{i}=*($ where $\hat{z}$ means delete $z$ ). We set

$$
F_{q} \xi^{l}(n)=\coprod_{j=0}^{q} \Xi_{j}^{l}(n) / \approx \quad \text { and } \quad D_{q} \xi^{l}(n)=F_{q} \xi^{l}(n) / F_{q-1} \xi^{l}(n) .
$$

Then $\xi^{l}(n)$ is unstably homotopy equivalent to $W^{l}(n)$ such that there is a stable homotopy equivalence $\xi^{l}(n) \underset{s}{\simeq} \bigvee_{1 \leq q} D_{q} \xi^{l}(n)$.

We construct an unstable map

$$
\vartheta_{q, n}^{l}: \Xi_{q}^{l}(n) \rightarrow P_{q n, n}^{l}(\mathbf{C}) .
$$

We identify

$$
D^{2 n-2}=\left\{f=z^{n}+0 \cdot z^{n-1}+a_{1} z^{n-2}+\cdots+a_{n-1}: a_{i} \in \mathrm{C}, \sum_{i=1}^{n-1}\left|a_{i}\right|^{2} \leq 1\right\} .
$$

A little 2-cube $c$ naturally defines a map $\tilde{c}: D^{2 n-2} \rightarrow \mathrm{C}^{n}$ (where we identify $\mathrm{C}^{n}$ with the space of monic polynomials over $\mathrm{C}$ of degree $n$ ) as follows. We fix a homeomorphism $h: \mathrm{C} \cong J^{2}$, where $J=(0,1)$. For $f=\prod_{i=1}^{n}\left(z-\alpha_{i}\right) \in$ $D^{2 n-2}$, we set

$$
\widetilde{c}(f)=\prod_{i=1}^{n}\left(z-c \circ h\left(\alpha_{i}\right)\right) \in \mathrm{C}^{n} .
$$


Now we set

$$
\vartheta_{q, n}^{l}\left(\left\langle c_{1}, \ldots, c_{q}\right\rangle, f_{1}, \ldots, f_{q}\right)=\prod_{i=1}^{q} \widetilde{c}_{i}\left(f_{i}\right),
$$

where the right-hand side is a multiplication of polynomials. Note that for $i \neq j, \widetilde{c}_{i}\left(f_{i}\right)$ and $\widetilde{c}_{j}\left(f_{j}\right)$ do not have a common root. Since at most $l$ of $f_{i}$ is of the form $z^{n}, \vartheta_{k, n}^{l}$ is indeed a map to $P_{q n, n}^{l}(\mathrm{C})$.

REMARK. Let $f=\prod_{j=1}^{q n}\left(z-\alpha_{j}\right) \in \operatorname{Im} \vartheta_{q, n}^{l}$. Then, by the definition of $\vartheta_{q, n}^{l}$, we have $\alpha_{j} \in J^{2}(1 \leq j \leq q n)$. We use this fact in Section 6 .

Now we define a stable map $f_{k, n}^{l}(\mathrm{C}): \bigvee_{q=1}^{k} D_{q} \xi^{l}(n) \rightarrow P_{k n, n}^{l}(\mathrm{C})$ to be the following composite of maps:

$$
f_{k, n}^{l}(\mathrm{C}): \bigvee_{q=1}^{k} D_{q} \xi^{l}(n) \stackrel{\vee e_{q, n}^{l}}{\longrightarrow} \bigvee_{q=1}^{k} \Xi_{q}^{l}(n) \stackrel{\vee \vartheta_{q, n}^{l}}{\longrightarrow} \bigvee_{q=1}^{k} P_{q n, n}^{l}(\mathrm{C}) \stackrel{j}{\longrightarrow} P_{k n, n}^{l}(\mathrm{C}),
$$

where $e_{q, n}^{l}: D_{q} \xi^{l}(n) \rightarrow \Xi_{q}^{l}(n)$ is a generalization of the Snaith stable splitting map and $j$ is the map induced from inclusions $P_{q n, n}^{l}(\mathrm{C}) \hookrightarrow P_{k n, n}^{l}(\mathrm{C})$.

(C) We have a stable homotopy equivalence $P_{k n, n}^{0}(\mathrm{C}) \underset{s}{\simeq} X_{k, n}^{0}(\mathrm{C})$ without specifying a map. (Compare (1.1).) From the construction of the map $f_{k, n}^{l}(\mathrm{C})$ in (5.2), it is easy to see that $f_{k, n}^{0}(\mathrm{C})$ indeed gives a stable homotopy equivalence.

(D) The initial step for induction is proved in (C).

LEMMA 5.3. There is a commutative diagram

$$
\begin{aligned}
& \cdots \longrightarrow H_{*}\left(X_{k, n}^{l-1}(\mathrm{C})\right) \stackrel{i_{*}}{\longrightarrow} H_{*}\left(X_{k, n}^{l}(\mathrm{C})\right) \longrightarrow H_{*-2 l(n-1)}\left(X_{k-l, n}^{0}(\mathrm{C})\right) \\
& f_{k, n}^{l-1}(\mathrm{C})_{*} \downarrow \quad f_{k, n}^{l}(\mathrm{C})_{*} \downarrow \quad f_{k-l, n}^{0}(\mathrm{C})_{*} \downarrow \\
& \cdots \longrightarrow H_{*}\left(P_{k n, n}^{l-1}(\mathrm{C})\right) \stackrel{i_{*}}{\longrightarrow} H_{*}\left(P_{k n, n}^{l}(\mathrm{C})\right) \longrightarrow H_{*-2 l(n-1)}\left(P_{(k-l) n, n}^{0}(\mathrm{C})\right) \\
& \longrightarrow H_{*-1}\left(X_{k, n}^{l-1}(\mathrm{C})\right) \longrightarrow \cdots \\
& f_{k, n}^{l-1}(\mathbf{C})_{*} \downarrow \\
& \longrightarrow H_{*-1}\left(P_{k n, n}^{l-1}(\mathrm{C})\right) \longrightarrow \cdots
\end{aligned}
$$

where each row is exact and $i$ are suitable inclusions.

Proof. The first row is similar to (4.6) and is proved in [10, Propositions $4.5,5.4]$. The second row can be proved similarly using the fact that

$$
P_{k n, n}^{l}(\mathrm{C})-P_{k n, n}^{l-1}(\mathrm{C})=\mathrm{SP}^{l}(\mathrm{C}) \times P_{(k-l) n, n}^{0}(\mathrm{C}) .
$$


From the construction of the stable map $f_{k, n}^{l}(\mathrm{C})$ in (5.2), the diagram of the lemma is commutative. This completes the proof of Lemma 5.3.

Now using Lemma 5.3, we can prove inductively that $f_{k, n}^{l}(\mathrm{C})$ induces an isomorphism in homology, hence is a stable homotopy equivalence. This completes the proof of Theorem A (i) for $F=\mathrm{C}$.

Proof of Theorem C. Hereafter we assume $(n, l) \neq(2,0)$. By a similar argument to the construction of the unstable map $\vartheta_{q, n}^{l}$ in (5.1), we construct an unstable map

$$
\varrho_{q, n}^{l}: \Xi_{q}^{l}(n) \rightarrow X_{q, n}^{l}(\mathrm{C})
$$

as follows. We identify

$$
D^{2 n-2}=\left\{g=\left(z, z-\alpha_{1}, \ldots, z-\alpha_{n-1}\right): \alpha_{i} \in \mathrm{C}, \sum_{i=1}^{n-1}\left|\alpha_{i}\right|^{2} \leq 1\right\} .
$$

A little 2-cube $c$ naturally defines a map $\tilde{c}: D^{2 n-2} \rightarrow C^{n}$ as follows. For $g=\left(z, z-\alpha_{1}, \ldots, z-\alpha_{n-1}\right) \in D^{2 n-2}$, we set

$$
\widetilde{c}(g)=\left(z-c \circ h(0), z-c \circ h\left(\alpha_{1}\right), \ldots, z-c \circ h\left(\alpha_{n-1}\right)\right) \in \mathrm{C}^{n} .
$$

Then we set

$$
\varrho_{q, n}^{l}\left(\left\langle c_{1}, \ldots, c_{q}\right\rangle, g_{1}, \ldots, g_{q}\right)=\mu\left(\widetilde{c}_{1}\left(g_{1}\right), \ldots, \widetilde{c}_{q}\left(g_{q}\right)\right),
$$

where the right-hand side means componentwise multiplication of $n$-tuples of polynomials. Since at most $l$ of $g_{i}$ is of the form $(z, \ldots, z), \varrho_{k, n}^{l}$ is indeed a map to $X_{q, n}^{l}(\mathrm{C})$.

Since $P_{k, n}^{l}(\mathrm{C})$ and $X_{k, n}^{l}(\mathrm{C})$ are simply connected, the inclusion of Step (A) is in fact a homotopy equivalence. Hence, Theorem $\mathrm{C}$ follows from the following:

Proposition 5.4. (i) There are unstable maps $H_{k, n}^{l}: F_{k} \xi^{l}(n) \rightarrow P_{k n, n}^{l}(\mathrm{C})$ and $\widetilde{H}_{k, n}^{l}: F_{k} \xi^{l}(n) \rightarrow X_{k, n}^{l}(\mathrm{C})$ so that the following diagrams are homotopy commutative:

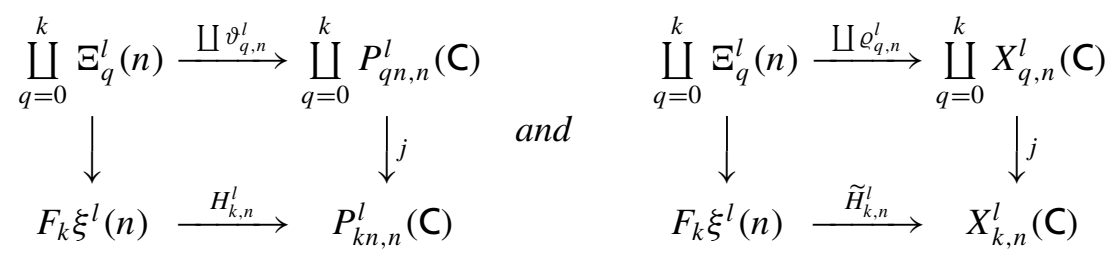

(ii) The maps $H_{k, n}^{l}$ and $\widetilde{H}_{k, n}^{l}$ are homotopy equivalences. 
Proof. (i) The right diagram for $l=0$ and $n \geq 3$ is Theorem 1.4 in [7]. By a similar argument, we can prove (i).

(ii) By Theorem A (i) for $F=\mathrm{C}$ and Theorem B (i), $H_{k, n}^{l}$ and $\widetilde{H}_{k, n}^{l}$ induce isomorphisms in homology. Since the both sides of $H_{k, n}^{l}$ and $\widetilde{H}_{k, n}^{l, n}$ are simply connected, (ii) follows. This completes the proof of Proposition 5.4, and, consequently, of Theorem C.

\section{Proofs of Theorem A (i) for $F=R$ and (ii)}

We follow Steps (A)-(D) in Section 5.

(A) By a similar argument to the repetition theorem in [1, p. 42], it is easy to show that (A) holds for $P_{k n+j, n}^{l}(\mathrm{R})$ and $Q_{k n+j, n}^{l}(F)(0 \leq j \leq n-1)$.

(B) Since the arguments are similar, we construct $g_{k, n}^{2 i}(\mathrm{R})$ and give remarks for the other cases. Similarly to $\eta_{q, i, n}$ in (4.3), there is an inclusion $v_{q, n}^{i}$ : $\operatorname{Im} \vartheta_{q, n}^{i} \rightarrow Q_{2 q n, n}^{2 i}(\mathrm{R})$ defined as follows. Let $f=\prod_{j=1}^{q n}\left(z-\alpha_{j}\right) \in \operatorname{Im} \vartheta_{q, n}^{i}$. By Remark after (5.1), we have $\alpha_{j} \in J^{2}(1 \leq j \leq q n)$. We define $v_{q, n}^{i}(f)$ to be the polynomial whose roots are $\alpha_{j}$ and $\bar{\alpha}_{j}(1 \leq j \leq q n)$. Using this, we have a stable map

$$
v_{q, n}^{i} \circ \vartheta_{q, n}^{i} \circ e_{q, n}^{i}: D_{q} \xi^{i}(n) \rightarrow Q_{2 q n, n}^{2 i}(\mathrm{R}) .
$$

By a similar argument to (4.10), the fact $Q_{n, n}^{0}(\mathrm{R})=P_{n, n}^{0}(\mathrm{R}) \simeq S^{n-2}$ (compare Example 2.2 (i)) gives a stable map $\Sigma^{p(n-2)} D_{q} \xi^{i}(n) \rightarrow Q_{(p+2 q) n, n}^{2 i}(\mathrm{R})$. This map naturally defines $g_{k, n}^{2 i}(\mathrm{R})$.

In order to construct $f_{k, n}^{2 i}(\mathrm{R})$, it is convenient to use (4.7), and in order to construct $g_{k, n}^{l}(\mathrm{C})$, note that the map $f_{k, n}^{l}(\mathrm{C})$ in (5.2) is in fact a map to $Q_{k n, n}^{l}(\mathrm{C})$.

(C) In order to prove that $f_{k, n}^{0}(\mathrm{R})$ is a stable homotopy equivalence, it suffices to prove that

$$
P_{k n, n}^{0}(\mathrm{R}) \underset{s}{\simeq} X_{k, n}^{0}(\mathrm{R})
$$

without specifying a map. This is proved in the same way as in the proof of $P_{k n, n}^{0}(\mathrm{C}) \underset{s}{\simeq} X_{k, n}^{0}(\mathrm{C})$ in [14, p. 151] and an outline is as follows. Let $\bar{\Sigma}_{1}$ and $\bar{\Sigma}_{2}$ be the complements of $P_{k n, n}^{0}(\mathrm{R})$ and $X_{k, n}^{0}(\mathrm{R})$ in $S^{k n}=\mathrm{R}^{k n} \cup\{\infty\}$, respectively. Recall that in Lemma 4.4, we discussed the spectral sequence $E^{r}\left(G\left(\bar{\Sigma}_{2}\right)\right)$ which converges to $H_{*}\left(G\left(\bar{\Sigma}_{2}\right)\right)$. Similarly, we can consider the spectral sequence $E^{r}\left(G\left(\bar{\Sigma}_{1}\right)\right)$. We see that $E^{1}\left(G\left(\bar{\Sigma}_{1}\right)\right) \cong E^{1}\left(G\left(\bar{\Sigma}_{2}\right)\right)$. Then by the same argument as in [14, p. 151], we can prove that $G\left(\bar{\Sigma}_{1}\right) \underset{s}{\simeq} G\left(\bar{\Sigma}_{2}\right)$. Since $G\left(\bar{\Sigma}_{i}\right) \simeq \bar{\Sigma}_{i}$ and the Spanier-Whitehead duals of stably homotopy equivalent spaces are stably homotopy equivalent, we have $P_{k n, n}^{0}(\mathrm{R}) \simeq X_{k, n}^{0}(\mathrm{R})$. 
This completes the proof of (6.1), and hence $f_{k, n}^{0}(\mathrm{R})$ is a stable homotopy equivalence.

Since $f_{k, n}^{0}(F)=g_{k, n}^{0}(F), g_{k, n}^{0}(F)$ is also a stable homotopy equivalence.

(D) The initial step for induction for Theorem A for $F=\mathrm{R}$ is proved in (6.1). Similar diagrams to Lemma 5.3 hold. For example, in order to prove Theorem A (i) for $F=\mathrm{R}$, we substitute (4.6) for the first row of Lemma 5.3. Then we can prove inductively that $f_{k, n}^{l}(\mathrm{R})$ and $g_{k, n}^{l}(F)$ are stably homotopy equivalent.

\section{REFERENCES}

1. Arnold, V. I., On some topological invariants of algebraic functions, Trudy Moscov. Mat. Obshch. 21 (1970), 27-46; English transl. in Trans. Moscow Math. Soc. 21 (1970), 30-52.

2. Boyer, C. P., and Mann, B. M., Monopoles, non-linear $\sigma$ models, and two-fold loop spaces, Comm. Math. Phys. 115 (1988), 571-594.

3. Brown, E. H., and Peterson, F. P., On the stable decomposition of $\Omega^{2} S^{r+2}$, Trans. Amer. Math. Soc. 243 (1978), 287-298.

4. Cohen, F. R., Cohen, R. L., Mann, B. M., and Milgram, R. J., The topology of rational functions and divisors of surfaces, Acta Math. 166 (1991), 163-221.

5. Cohen, F. R., Cohen, R. L., Mann, B. M., and Milgram, R. J., The homotopy type of rational functions, Math. Z. 213 (1993), 37-47.

6. Cohen, F. R., Lada, T. J., and May, J. P., The Homology of Iterated Loop Spaces, Lecture Notes in Math. 533, Springer, Berlin and New York (1976).

7. Cohen, R. L., and Shimamoto, D. H., Rational functions, labelled configurations, and Hilbert schemes, J. London Math. Soc. 43 (1991), 509-528.

8. Fuks, D. B., Cohomology of the braid group mod 2, Funktsional. Anal. i Prilozhen. 4 (1970), 62-73; English transl. in Functional Anal. Appl. 4 (1970), 143-151.

9. Guest, M. A., Kozlowski, A., and Yamaguchi, K., Stable splitting of the space of polynomials with roots of bounded multiplicity, J. Math. Kyoto Univ. 38 (1998), 351-366.

10. Kamiyama, Y., Polynomial model for homotopy fibers associated with the James construction, Math. Z. 237 (2001), 149-180.

11. Kozlowski, A., and Yamaguchi, K., Topology of complements of discriminants and resultants, J. Math. Soc. Japan 52 (2000), 949-959.

12. Mostovoy, J., Spaces of rational loops on a real projective space, Trans. Amer. Math. Soc. 353 (2001), 1959-1970.

13. Segal, G. B., The topology of spaces of rational functions, Acta Math. 143 (1979), 39-72.

14. Vassiliev, V. A., Complements of Discriminants of Smooth Maps: Topology and Applications. Revised ed., Transl. of Math. Monographs 98, Amer. Math. Soc., Providence, RI (1994).

DEPARTMENT OF MATHEMATICS

UNIVERSITY OF THE RYUKYUS

NISHIHARA-CHO

OKINAWA 903-0213

JAPAN

E-mail: kamiyama@sci.u-ryukyu.ac.jp 